\title{
Circuit Distortion Analysis Based on the Simplified Newton's Method
}

\author{
M. M. Gourary, ${ }^{1}$ S. G. Rusakov, ${ }^{1}$ S. L. Ulyanov, ${ }^{1}$ M. M. Zharov, $^{1}$ and B. J. Mulvaney ${ }^{2}$ \\ ${ }^{1}$ IPPM, Russian Academy of Sciences, Sovetskaya 3, Moscow 124365, Russia \\ ${ }^{2}$ Freescale Semiconductor Inc., 7700 W. Parmer Lane, Austin, TX, USA
}

Correspondence should be addressed to S. L. Ulyanov, ulyas@ippm.ru

Received 31 March 2011; Accepted 13 June 2011

Academic Editor: Muhammad Taher Abuelma'atti

Copyright ( $) 2011$ M. M. Gourary et al. This is an open access article distributed under the Creative Commons Attribution License, which permits unrestricted use, distribution, and reproduction in any medium, provided the original work is properly cited.

\begin{abstract}
A new computational technique for distortion analysis of nonlinear circuits is presented. The new technique is applicable to the same class of circuits, namely, weakly nonlinear and time-varying circuits, as the periodic Volterra series. However, unlike the Volterra series, it does not require the computation of the second and third derivatives of device models. The new method is computationally efficient compared with a complete multitone nonlinear steady-state analysis such as harmonic balance. Moreover, the new technique naturally allows computing and characterizing the contributions of individual circuit components to the overall circuit distortion. This paper presents the theory of the new technique, a discussion of the numerical aspects, and numerical results.
\end{abstract}

\section{Introduction}

RF circuits are generally designed to be linear with respect to the signal path. However, the desired signal may be weakly distorted due to nonlinearities of the circuit components. Analyzing this nonlinear distortion is an important problem in the design of RF circuits $[1,2]$. Another important practical task is the computation of distortion contributions due to individual nonlinearities of the circuit. This information is essential to determine critical circuit elements and improve the design.

The traditional approach to measuring distortion is to apply one or more pure test tones to the circuit's input and determine harmonics or intermodulation products at the output $[3,4]$. This approach is applied to both time-invariant circuits (such as amplifiers) and circuits with periodic excitation (such as mixers).

Consequently, distortion characteristics can be obtained using quasiperiodic steady-state nonlinear analysis such as harmonic balance technique [5-11] or mixed frequency time method $[5,12]$. Such methods can be applied to circuits with strong nonlinear behavior but require essential computational efforts. Also these methods do not provide the computation of individual distortion contributions.
For distortion analysis of time-invariant weakly nonlinear circuits, the approach based on Volterra series is more efficient [3, 4, 13-15]. According to this approach, each nonlinearity in the circuit is presented by a power series expansion up to the third order, and each term of expansion is associated with a source. The analysis leads to three linear systems, one for each order of nonlinear circuit response, that are solved successively beginning with the first order. This analysis implemented in Spice simulators (e.g., the DISTO analysis) provides the distortion characterization after the computation of DC operating point.

The essential disadvantage of this approach is the necessity to compute power series coefficients for each nonlinearity in the circuit. This requires that the simulator has explicitly coded second- and third-order derivatives of the device models. With modern device models, which include nonlinear dependencies in several variables as well as different model behavior in different regions of device operation, computation of high-order derivatives is extremely difficult, if not impossible. Also this requirement limits the introduction of new models that may prevent the distortion analysis with a wide class of behavioral models.

In comparison with conventional nonlinear distortion analysis based on computing distortion about the DC 
operating point, distortion analysis of communication circuits often requires determining the distortion about a periodically time-varying operating point. In this case, the input signal is considered as a small excursion about the periodically varying operating point, and the distortion characteristics can be obtained from the simulation of weakly nonlinear behavior with respect to the input signal [16]. Thus, these circuits must be considered as periodically timevarying systems. Conventional distortion analysis can be extended to periodically time-varying nonlinear circuits using time varying Volterra series [17]. However, the same difficulty with device models persists.

Thus, the introduction of an efficient special-purpose distortion mode into circuit simulators is a current challenge in $\mathrm{RF}$ and microwave CAD engineering.

We focus on the following practical problems for distortion analysis. First, in order to avoid the analytic computation of second and third derivatives of device models, we need an alternative to the Volterra series technique that retains the same overall accuracy. Second, we need to handle the problem of distortion of periodic time-varying circuits. The third practical problem we wish to address is the computation of the individual contributions of each component of the circuits to the overall distortion. This of course has important applications in guiding circuit design. The Volterra series technique supports such computation, so we need to retain this capability.

This paper discusses a new approach for the distortion analysis [18] based on the simplified Newton's method [19]. The computational efforts of the new approach are practically the same as for distortion analysis based on Volterra series, and radically lower in comparison with complete multitone nonlinear steady-state analysis. The new approach supports the computation of individual distortion contributions. A matrix form for the characterization of individual contributions is proposed that is well suited for circuit simulators, and the computational technique for extracting individual contributions is presented.

It is important to note that the proposed approach does not require computing high-order derivatives of device model nonlinearities, and thus there is no need to code the second and third derivatives for all the device models. This approach provides basically the same order of accuracy as the Volterra series, due to properties of the simplified Newton's method. The technique can be readily implemented in a generalpurpose circuit simulator.

A similar approach to distortion analysis has been proposed [20] which is based on linear-centric models and successive chord method. This likewise avoids the computation of second and third derivatives of device models but differs from the presented technique in computation of third-order and higher-distortion components, essential for determining the IM3 metric. The approach does not take into account the interaction of nonlinearities in the computation of distortion contributions.

The paper is organized as follows. Section 2 describes the formulation of the new approach for distortion analysis and presents the basic computational procedure. Section 3 discusses the application of this approach to the periodic distortion analysis of nonlinear circuits in the frequency domain in the framework of the harmonic balance method. Section 4 is devoted to the problem of computing individual contributions. Examples of computing distortion metrics and individual contributions for some typical circuits are given in Section 5.

\section{Formulation of the Approach}

2.1. Basic Model for Distortion Analysis. The distortion analysis is intended to provide a measure of the distortion products when one or more pure sinusoids are applied to the input of the circuit. In RF circuits, there may be an extra periodic excitation that determines the periodically varying operating point.

In this case, the nonlinear circuit can be described by a system of differential-algebraic equations $[1,5]$

$$
\frac{d}{d t} q(x(t))+i(x(t))+\tilde{u}(t)+u(t)=0,
$$

where $q$ is the vector of charges, $x$ is the vectors of nodal voltages, $\tilde{u}(t)$ is a large periodic excitation with frequency $\tilde{\omega}$, and $u(t)=u_{1}(t)+u_{2}(t)+\cdots+u_{M}(t)$ is a vector of small periodic excitations corresponding to the set of independent sources with incommensurate frequencies $\omega_{i}, i=1, \ldots, M$.

Equation (1) corresponds to the problem of distortion analysis for periodically time-varying circuits. The basic model for conventional distortion analysis about DC operating point can be obtained as the particular case of (1) with $\tilde{u}(t)=0$, that is,

$$
\frac{d}{d t} q(x(t))+i(x(t))+u(t)=0 .
$$

2.2. Features of Volterra Series-Based Technique. The purpose of the nonlinear distortion analysis is the evaluation of the deviation of the circuit behavior from the desired linear behavior. There are effective methods to provide this evaluation, such as Volterra series [13,15] or alternative method of direct calculation of nonlinear responses [3].

These methods present the approximate solution of (1) or (2) in the form

$$
x(t) \approx x^{(n)}(t)=\tilde{x}(t)+\Delta x^{(0)}(t)+\cdots+\Delta x^{(n-1)}(t),
$$

where $\tilde{x}(t)$ is the periodic solution without small excitations, $\Delta x^{(0)}(t)$ is the linear response of the system, that is, the result of periodic AC analysis [1], and $\Delta x^{(k)}(t)(k>0)$ is the deviation of $(k+1)$ th order with respect to the norm of small excitations $\left\|\Delta x^{(k)}(t)\right\|=O\left(\|u(t)\|^{k+1}\right)$. Here, the Landau symbol $O$ means the asymptotic order.

Each deviation is computed by solving the linear system in the frequency domain. The matrices of linear systems are obtained using linear AC analysis. The rhs vectors (equivalent current sources) are obtained by the computation of polynomials of previous-order deviations. The polynomials coefficients are defined by Taylor expansion of voltagecurrent and voltage-charge characteristics of circuit devices. 
The accuracy of the approximate solution (3) is characterized by the expression

$$
\left\|x^{(n)}(t)-x(t)\right\|=O\left(\|u(t)\|^{n+1}\right) .
$$

Usually it is assumed that nonlinearities are sufficiently small to neglect terms higher than third order. Thus, the analysis leads to successively solving the set of linear systems for each order beginning from the first order. The firstorder solution $x^{(1)}(t)$ is then used to calculate the equivalent current sources to determine the second-order solution $x^{(2)}(t)$. Both the first- and second-order solutions are used to compute the third-order solution $x^{(3)}(t)$. Thus, only three steps are needed to obtain third-order solution.

The main advantage of Volterra series in the comparison with complete nonlinear analysis is the reduction of computational efforts. This results in a much smaller dimension of the small signal linear systems in the comparison with the dimension of the linear system solved at the iteration step of nonlinear analysis.

The essential disadvantage of the Volterra series is the necessity of computing high-order power series coefficients for each nonlinearity in component models. This requirement limits the introduction of new device models because it leads to the very complex practical problem of analytically deriving high-order derivatives of the models.

2.3. Foundation of Computational Procedure Based on Simplified Newton's Method. Below, we discuss the new approach that provides the similar accuracy (4) by the solving of the same number of linear systems. But unlike the Volterra series, this approach does not require complicated procedures to evaluate residuals in terms of high-order coefficients of Taylor expansions of circuit nonlinearities.

Firstly, we can point out that any computational method to determine steady-state solution of (1) reduces the problem to the system of nonlinear algebraic equations

$$
f(z)=w,
$$

where $w, z$ are $N$-dimensional vectors of input and internal signals in frequency or time domain. Let the vector $z^{(0)}$ be a solution of the problem obtained from (5) by neglecting small excitations, that is,

$$
f\left(z^{(0)}\right)=0
$$

The nonlinear algebraic equation (5) are typically solved by the Newton's method [19]

$$
\begin{array}{r}
J\left(z^{(k)}\right) \Delta z^{(k)}=w-f\left(z^{(k)}\right), \quad z^{(k+1)}=z^{(k)}+\Delta z^{(k)}, \\
k=0,1,2, \ldots,
\end{array}
$$

where

$$
J\left(z^{(k)}\right)=\left[\begin{array}{ccc}
\frac{\partial f_{1}\left(z^{(k)}\right)}{\partial z_{1}} & \cdots & \frac{\partial f_{1}\left(z^{(k)}\right)}{\partial z_{N}} \\
\vdots & \ddots & \vdots \\
\frac{\partial f_{N}\left(z^{(k)}\right)}{\partial z_{1}} & \cdots & \frac{\partial f_{N}\left(z^{(k)}\right)}{\partial z_{N}}
\end{array}\right]
$$

is the Jacobian matrix of the system (5), and $\Delta z^{(k)}$ is the vector of corrections.

Here, we apply the simplified Newton's method [19] that is similar to the Newton's method (7) except that the Jacobian matrix is kept fixed and equal to $J\left(z^{(0)}\right)$. Thus, the method does not require any update of the Jacobian matrix.

The numerical scheme contains one Newton step and $(k-1)$ steps of the simplified Newton's method. For our consideration, it is important that the reduction of error can be estimated as follows $[19,21]$ :

$$
\left\|z^{(k)}-z^{*}\right\|=O\left(\left\|z^{(0)}-z^{*}\right\|^{k+1}\right), \quad k=1,2, \ldots,
$$

where $z^{*}$ is the exact solution.

Using the estimate $\left\|z^{(0)}-z^{*}\right\|=O(\|w\|)$, we have from (9) for $k=n$ that

$$
\left\|z^{(n)}-z^{*}\right\|=O\left(\|w\|^{n+1}\right) .
$$

Thus, the simplified Newton's method provides the same accuracy order as the Volterra series method (4) after the same number of steps and under the same limitations on the level of input amplitude. Hence, three steps of the method (7) are sufficient to provide the third-order solution.

To obtain corrections $\Delta z^{(k)}$ up to the third order, the computational procedure after the determination of the initial guess by (6) includes the following three steps:

(1)

$$
J\left(z^{(0)}\right) \Delta z^{(0)}=w
$$

$$
J\left(z^{(0)}\right) \Delta z^{(1)}=w-f\left(z^{(1)}\right),
$$

$$
J\left(z^{(0)}\right) \Delta z^{(2)}=w-f\left(z^{(2)}\right) .
$$

Step 1 of this technique coincides with the Volterra series method while steps 2 and 3 differ by the evaluation of rhs vectors. Rather than expansion of nonlinear functions in power series (forming equivalent current sources), the rhs vectors for systems (12), (13) are computed by the same technique as nonlinear analysis, described in Section 3. As a result, the new approach avoids the computation of Taylor expansions of circuit nonlinearities.

2.4. Numerical Aspects. One shortcoming of the approach is connected with the possible growth of numerical noise in (12), (13) for cases of small deviations $\Delta z^{(0)}, \Delta z^{(1)}$. This is particularly critical for step 3 . To decrease this effect, we propose another form for the rhs vector (13).

The first-order Taylor expansion of $f\left(z^{(2)}\right)$ yields

$$
\begin{aligned}
f\left(z^{(2)}\right) & =f\left(z^{(1)}+\Delta z^{(1)}\right) \\
& =f\left(z^{(1)}\right)+J\left(z^{(1)}\right) \Delta z^{(1)}+O\left(\left\|\Delta z^{(1)}\right\|^{2}\right) .
\end{aligned}
$$


Taking into account that $\left\|\Delta z^{(1)}\right\|=O\left(\|w\|^{2}\right)$, we can evaluate $O\left(\left\|\Delta z^{(1)}\right\|^{2}\right)=O\left(\|w\|^{4}\right)$. So neglecting this term does not change the order of error at step 3 (see (10) for $n=3$ ). The substitution (14) into (13) leads to the modified expression of step 3

$$
J\left(z^{(0)}\right) \Delta z^{(2)}=w-f\left(z^{(1)}\right)-J\left(z^{(1)}\right) \Delta z^{(1)} .
$$

Subtracting (12) from (15), a more convenient form of this expression is obtained

$$
J\left(z^{(0)}\right) \Delta z^{(2)}=\left[J\left(z^{(0)}\right)-J\left(z^{(1)}\right)\right] \Delta z^{(1)} .
$$

In many cases, it is efficient to perform step 2 by the equation

$$
J\left(z^{(0)}\right) \Delta z^{(1)}=w-\varphi,
$$

where vector $\varphi$ approximates $f\left(z^{(1)}\right)$ with the accuracy

$$
\left\|\varphi-f\left(z^{(1)}\right)\right\|=O\left(\|w\|^{3}\right) .
$$

If step 2 is performed by (17) instead of (12), then previous considerations lead to the following expression instead of (16):

$$
J\left(z^{(0)}\right) \Delta z^{(2)}=\left[J\left(z^{(0)}\right)-J\left(z^{(1)}\right)\right] \Delta z^{(1)}+\varphi-f\left(z^{(1)}\right) .
$$

Expression (16) is useful for the analysis of contributions. Expressions (17), (19) will be used in Section 3 to reduce the number of linear systems to be solved.

\section{Distortion Analysis in Frequency Domain}

3.1. Distortion Analysis of Periodic Circuits. We now apply the simplified Newton's method described in Section 2 to compute distortion of a periodic circuit simulated with the harmonic balance method. This can be used in place of a multitone harmonic balance simulation in situations where some of the driving signals are small and subject to mild nonlinearity. The quasiperiodic harmonic balance (HB) method [5] transforms (1) to the following nonlinear algebraic system in the frequency domain

$$
H(X)=I(X)+\Omega \cdot Q(X)+\widetilde{U}+\sum_{i=1}^{M} U_{i}=0,
$$

where vectors $X, I(X), Q(X), \tilde{U}$, and $U$ are frequency domain representation of vectors $x(t), i(x), q(x), \widetilde{u}(t)$, and $u(t)$, and $\Omega$ is a block-diagonal matrix of combinational frequencies of fundamentals $\widetilde{\omega}, \omega_{i}$.

Solving the nonlinear system (20) by the Newton's method results in the linear system for corrections $\Delta X^{(k)}$,

$$
J^{\mathrm{HB}}\left(X^{(k)}\right) \Delta X^{(k)}=-H\left(X^{(k)}\right),
$$

where $J^{\mathrm{HB}}$ is the multitone harmonic Jacobian matrix [5].

The vector of unknowns contains all harmonics of the linear combinations with integer coefficients of all fundamentals $\omega=\tilde{k} \widetilde{\omega}+\nu$, where $v$ denotes the small signal combinational frequency of the form

$$
\nu=k_{1} \cdot \omega_{1}+k_{2} \cdot \omega_{2}+\cdots+k_{M} \cdot \omega_{M} .
$$

The number of complex unknowns in (20) or (21) is equal to $N \cdot(2 \widetilde{K} \cdot K+\widetilde{K}+K+1)$, where $N$ is the circuit dimension, $\widetilde{K}$ is the number of large signal harmonics, and $K$ is the number of positive small signal combinational frequencies $v$.

Now, we consider the approximate solution of (20) by the simplified Newton's procedure (7) with fixed Jacobian matrix.

The initial step (6) is solving the reduced problem that is in our case a single tone $\mathrm{HB}$ problem

$$
\widetilde{H}(\tilde{X})=\tilde{I}(\tilde{X})+\widetilde{\Omega} \cdot \widetilde{Q}(\tilde{X})+\widetilde{U}=0 .
$$

The nonlinear system (23) is obtained from (20) by applying only the large signal excitation.

After determining the initial guess $X^{(0)}=\tilde{X}$, one can apply the iterative process (7) that for system (20) is presented in the form

$$
J^{\mathrm{HB}}(\tilde{X}) \Delta X^{(k)}=-H\left(X^{(k)}\right) .
$$

The Jacobian matrix $J^{\mathrm{HB}}(\tilde{X})$ corresponds to (20) linearized about the single tone steady-state solution, and the system (24) presents the problem of the periodic small signal analysis. This problem can be decomposed into the set of low dimensional problems [22] for each small signal combinational frequency (22)

$$
J^{\mathrm{SS}}\left(v_{j}\right) \Delta X^{(k)}\left(v_{j}\right)=-H^{(k)}\left(v_{j}\right) .
$$

The number of unknowns in each system (25) is equal to $N \cdot(2 \widetilde{K}+1)$, and there are $K$ systems with different $v_{j}$.

The entries of the matrix $J^{\mathrm{SS}}\left(v_{j}\right)$ can be defined by the Fourier transform of periodically varying conductance $G(t)$ and capacitance $C(t)$ matrices. But there is no need to obtain the small signal matrix in the frequency domain because systems (25) can be solved by efficient Krylov subspace methods with matrix-vector multiplication in the time domain [6].

Thus, each step of the simplified Newton's iterative process includes two numerical procedures:

(i) evaluation of the full rhs vector $H\left(X^{(k)}\right)$ by the same algorithm that is applied in the solving of multitone $\mathrm{HB}$ problem,

(ii) solving the linear systems (25) with rhs vectors defined by the frequency decomposition of the full rhs vector for the required small signal frequency set.

The evaluation of the full rhs vector for the modified step 3 (16) is performed by the corresponding $\mathrm{HB}$ expression

$$
H^{(2)}=\left[J^{\mathrm{HB}}\left(X^{(1)}\right)-J^{\mathrm{HB}}(\tilde{X})\right] \Delta X^{(1)},
$$

where $J^{\mathrm{HB}}\left(X^{(1)}\right)$ is harmonic Jacobian matrix (21) that is computed at the step 2 simultaneously with the computation of $H\left(X^{(1)}\right)$. 
3.2. The Numerical Scheme. Before proceeding with the distortion analysis, it is first necessary to determine the harmonics that must be considered in the computations.

The number of large signal harmonics is determined during the solving of the single-tone HB problem (23). To minimize the number of small signal harmonics that provides the accuracy order (10), we take into account the sinusoidal form of small input signals $u_{i}=U_{i} \exp \left( \pm j \omega_{i} t\right)$. In such case, the minimal sets of small signal combinational frequencies at the $n$th step $v^{(n)}=\left\{v_{1}^{(n)}, v_{2}^{(n)}, \ldots\right\}$ are defined by the following recurrent expressions:

$$
\begin{gathered}
v^{(1)}=\left\{\ldots, \omega_{i}, \ldots\right\}, \\
v^{(n+1)}=\left\{\ldots,\left|v_{j}^{(n)} \pm \omega_{i}\right|, \ldots\right\} .
\end{gathered}
$$

In the case of two small excitations, frequency sets (27) of second and third orders result in the forms

$$
\begin{aligned}
v^{(2)}= & \left\{0,2 \omega_{1}, 2 \omega_{2}, \omega_{1}+\omega_{2},\left|\omega_{1}-\omega_{2}\right|\right\}, \\
v^{(3)}= & \left\{\omega_{1}, \omega_{2}, 2 \omega_{1}+\omega_{2}, \omega_{1}+2 \omega_{2},\right. \\
& \left.\left|2 \omega_{1}-\omega_{2}\right|,\left|2 \omega_{2}-\omega_{1}\right|, 3 \omega_{1}, 3 \omega_{2}\right\} .
\end{aligned}
$$

Note that at the last step, it is sufficient to determine only those harmonics of (29) that are required by the user. For example, if only intermodulation distortion is needed, then frequency set (29) is reduced to only one frequency $v^{(3)}=$ $\left\{\left|2 \omega_{1}-\omega_{2}\right|\right\}$.

Small signal systems at step 2 are solved only for secondorder harmonics (28). It is equivalent to the performing of step 2 by (17), where vector $\varphi$ represents the rhs vector with zero third-order harmonics (29). So in this case, the third step must be performed by (19), that is, vector (26) must be added by the magnitudes of third-order harmonics obtained from the rhs vector of step $2, H^{(1)}\left(v_{j}^{(3)}\right)$.

The computational procedure includes the following steps.

Step 1 (Periodic large signal solution). Solve the system (23) by the single-tone $\mathrm{HB}$ method.

Save in memory the solution plus the conductance and capacitance matrices obtained at the last Newton step $\tilde{x}(t)$, $\widetilde{G}(t), \widetilde{C}(t)$.

Step 2. This step coincides with the standard periodic small signal analysis [23]. Here, the linear systems corresponding to the fundamentals $\omega_{1}, \omega_{2}$ are solved,

$$
\begin{aligned}
& J^{\mathrm{SS}}\left(\omega_{1}\right) \Delta X^{(0)}\left(\omega_{1}\right)=-U_{1}, \\
& J^{\mathrm{SS}}\left(\omega_{2}\right) \Delta X^{(0)}\left(\omega_{2}\right)=-U_{2} .
\end{aligned}
$$

Step 3. (a) Determine the first-order solution in time domain by applying inverse Fourier transform $\Gamma^{-1}$,

$$
x^{(1)}(t)=\tilde{x}(t)+\Gamma^{-1} \Delta X^{(0)} .
$$

(b) Compute time domain vectors of circuit charges, currents, and admittance matrices,

$$
q\left(x^{(1)}(t)\right), \quad i\left(x^{(1)}(t)\right), \quad G\left(x^{(1)}(t)\right), \quad C\left(x^{(1)}(t)\right) .
$$

(c) Compute the residual vector in the frequency domain by applying the Fourier transform $\Gamma$ and using (20),

$$
H\left(X^{(1)}\right)=\Gamma i\left(x^{(1)}(t)\right)+\Omega \Gamma q\left(x^{(1)}(t)\right)+\tilde{U}+U_{1}+U_{2} .
$$

(d) Decompose the vector $H\left(X^{(1)}\right)$ into $H^{(1)}\left(v_{j}^{(2)}\right)$ and $H^{(1)}\left(v_{j}^{(3)}\right)$.

(e) Determine corrections of solution by solving the systems

$$
J^{\mathrm{SS}}\left(v_{j}^{(2)}\right) \Delta X^{(1)}\left(v_{j}^{(2)}\right)=-H^{(1)}\left(v_{j}^{(2)}\right)
$$

for all of 5 small signal combinational frequencies $v_{j}^{(2)}$ from the set (28).

Step 4. (a) Transform the second-order correction to time domain by applying inverse Fourier transform

$$
\Delta x^{(1)}(t)=\Gamma^{-1} \Delta X^{(1)} .
$$

(b) Compute vector (26) by matrix-vector multiplication in time domain and Fourier transform to frequency domain

$$
\begin{aligned}
H^{(2)}= & \Gamma\left[\left(\widetilde{G}(t)-G\left(x^{(1)}(t)\right)\right) \Delta x^{(1)}(t)\right] \\
& +j \Omega \Gamma\left[\left(\widetilde{C}(t)-C\left(x^{(1)}(t)\right)\right) \Delta x^{(1)}(t)\right] .
\end{aligned}
$$

(c) Decompose vector $H^{(2)}$ into $H^{(2)}\left(v_{j}^{(3)}\right)$.

(d) Determine corrections by solving systems

$$
J^{\mathrm{SS}}\left(v_{j}^{(3)}\right) \Delta X^{(2)}\left(v_{j}^{(3)}\right)=-H^{(2)}\left(v_{j}^{(3)}\right)-H^{(1)}\left(v_{j}^{(3)}\right),
$$

for all required third-order harmonics from (29).

The conversion of signals from time to frequency domain and vise versa is performed using the multidimensional fast Fourier transform, which results in some aliasing error due to the finite number of sample points. Defining this number in accordance with the Nyquist frequency $(\geq 2 n+1)$, the aliasing error depends on harmonics higher than $n$. Thus, the required accuracy order (10) is provided for $n=3$ if the number of sample points is greater or equal to 7 for each small-signal fundamental.

3.3. DC Operating Point. The method for distortion analysis of weakly nonlinear circuits described by (2) can be obtained as a special case of the above method for periodically timevarying circuits.

In this case, the initial step of the analysis is determining the vector of DC solution. Thus, the vector $X^{(0)}$ contains $N$ nonzero components corresponding to the DC solution vector, and the $N \times N$ Jacobian matrix corresponds to the AC matrix $J^{\mathrm{SS}}(\nu)=G+j \nu C$, where matrices $G$ and $C$ are computed at the DC operating point. The computational procedure then follows the one presented above. 
3.4. Comparison with Linear-Centric Approach. The linearcentric approach for distortion analysis of time-varying and weakly nonlinear circuits has been described in [20]. The main distinction in the numerical strategy of the approach presented above in comparison with the linear-centric approach is the following: our numerical procedure includes the third step of the recursive process (11)-(13) in the form given by expression (19).

The linear-centric technique [20] also avoids the computation of high-order derivatives of circuit nonlinearities. The linear-centric model uses the successive chord iterative method, exploiting the constant Jacobian matrix for solving the nonlinear equations of harmonic balance, and requiring only one linear system solution to estimate distortion components.

It can be mentioned that this computational procedure is equivalent in practice to two first steps of our approach. However, as it follows from (10), two steps do not provide the desired accuracy of computations. In the general case, solving the linear problem (13) is required to compute third-order distortion component with required accuracy.

The following simple illustrative example demonstrates that the linear-centric model can lead to incorrect results due to limitations of number of solved linear problems.

Let a nonlinear resistor with voltage-current relation $I=V^{2}$ be excited by unit DC current source and a small sinusoidal signal. The corresponding equation has the form

$$
v^{2}-1-a \cdot \cos (\omega t)=0
$$

The DC solution of (38) is $v^{(0)}=1$.

The first-order correction is determined from the linear system obtained by linearization (38)

$$
2 \Delta v^{(0)}=a \cdot \cos (\omega t) .
$$

Therefore, the first-order solution is expressed as

$$
v^{(1)}=v^{(0)}+\Delta v^{(0)}=1+\frac{a}{2} \cdot \cos (\omega t) .
$$

The distortion contribution is determined from the linear system [20]

$$
2 \Delta v=-\left[\left(1+\frac{a}{2} \cdot \cos (\omega t)\right)^{2}-1-2 \cdot \frac{a}{2} \cdot \cos (\omega t)\right] .
$$

After elementary transformations, we have

$$
\Delta v=-\frac{a^{2}}{16}-\frac{a^{2}}{16} \cdot \cos (2 \omega t)
$$

Thus, we see that the voltage distortion does not contain third harmonics. Here, $\Delta v$ corresponds to the value $\Delta v^{(1)}$ from the recursive procedure.

For this simple example, it is easy to obtain the solution of (38) in the form

$$
v=\sqrt{1+a \cdot \cos (\omega t)} .
$$

This function contains third-order terms in its Taylor expansion $(y=a \cdot \cos (\omega t))$

$$
\sqrt{1+y}=1+\frac{y}{2}-\frac{y^{2}}{8}+\frac{y^{3}}{16}+O\left(y^{4}\right),
$$

which provide third harmonics. Note that if we perform the next step of our computational procedure, we obtain

$$
\Delta v^{(2)}=\frac{3 a^{3}}{64} \cos (\omega t)+\frac{a^{3}}{64} \cdot \cos (3 \omega t)+O\left(a^{4}\right) .
$$

This coincides with terms of the explicit Taylor expansion (44).

\section{Computation of Individual Contributions}

4.1. Contributions Characterization. An important problem in nonlinear distortion analysis is the computation of the contributions of each nonlinear component to the total circuit distortion. This information allows designers to determine which circuit elements are responsible for the distortion, thus providing guidance in meeting the required specifications.

Numerical characterization of contributions is to be achieved if one can evaluate the distortion metric in the vector form

$$
D=\left[D_{1}, D_{2}, \ldots, D_{m}, \ldots\right]
$$

with the following properties:

(1) $m$ th component of the vector depends on $m$ th nonlinearity and does not depend on any other nonlinearity,

(2) total distortion is the sum of all components of the vector

$$
D_{\text {total }}=\sum_{m} D_{m}
$$

However, for third-order distortion, this approach is incomplete because various nonlinearities can interact, and this interaction prevents different contributions from being associated with only one nonlinearity [3], as illustrated in the next example.

Consider the two-stage amplifier shown in Figure 1. Each stage of the amplifier is described by a transfer factor $(K)$ with small nonlinearities of second $(a)$ and third $(b)$ order

$$
\begin{aligned}
& v_{\text {out } 1}\left(v_{\text {inp } 1}\right)=K_{1} v_{\text {inp } 1}+a_{1} v_{\text {inp } 1}^{2}+b_{1} v_{\text {inp } 1}^{3} \\
& v_{\text {out } 2}\left(v_{\text {inp } 2}\right)=K_{2} v_{\text {inp } 2}+a_{2} v_{\text {inp } 2}^{2}+b_{2} v_{\text {inp2 }}^{3} .
\end{aligned}
$$

The circuit contains two nonlinearities defined by parameters $a_{1}, b_{1}$ for the first nonlinearity and $a_{2}, b_{2}$ for the second nonlinearity.

The output signal of the amplifier is obtained by

$$
v_{\text {out }}(v)=v_{\text {out } 2}\left(v_{\text {out } 1}(v)\right) .
$$

After substituting (48) into (49) and neglecting terms higher than third order, this expression can be presented in the form

$$
v_{\text {out }}(v)=v_{\text {out }}^{(1)}+v_{\text {out }}^{(2)}+v_{\text {out }}^{(3)},
$$




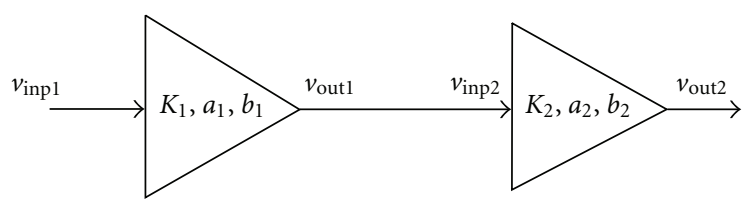

Figure 1: Two-stage amplifier.

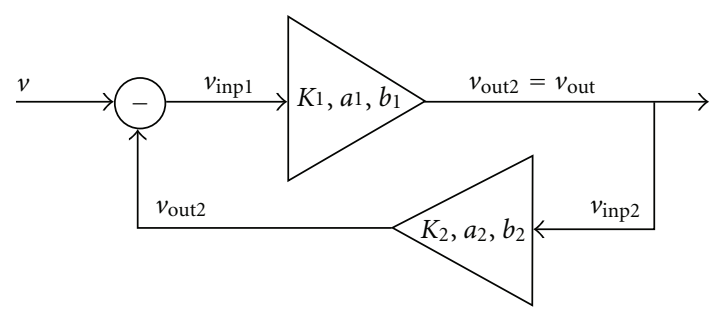

FIgUre 2: The amplifier with feedback loop.

where $v_{\text {out }}^{(1)}=K_{1} K_{2} v, v_{\text {out }}^{(2)}=K_{2} a_{1} v^{2}+K_{1}^{2} a_{2} v^{2}$, and $v_{\text {out }}^{(3)}=$ $K_{2} b_{1} v^{3}+K_{1}^{3} b_{2} v^{3}+2 K_{1} a_{1} a_{2} v^{3}$.

One can see that the expression for the second-order distortion $v_{\text {out }}^{(2)}$ is the sum of two terms, each of them corresponds to only one nonlinearity $\left(a_{1}\right.$ or $\left.a_{2}\right)$ and so can be considered as the contribution of this nonlinearity. Thus, the second-order contributions $D^{(2)}$ can be presented in the form (46) with $D_{1}^{(2)}=K_{2} a_{1} v^{2}, D_{2}^{(2)}=K_{1}^{2} a_{2} v^{2}$.

In contrast, the expression for the third-order distortion contains not only terms corresponding to each nonlinearity $\left(K_{2} b_{1} v^{3}, K_{1}^{3} b_{2} v^{3}\right)$ but also the term $2 K_{1} a_{1} a_{2} v^{3}$ depending on both nonlinearities.

Hence, the influences of circuit nonlinearities on the third-order distortion can be presented as a matrix of contributions

$$
D^{(3)}=\left[\begin{array}{cc}
D_{11}^{(3)} & 0 \\
D_{21}^{(3)} & D_{22}^{(3)}
\end{array}\right],
$$

where $D_{11}^{(3)}=K_{2} b_{1} v^{3}$, and $D_{22}^{(3)}=K_{1}^{3} b_{2} v^{3}, D_{21}^{(3)}=2 K_{1} a_{1} a_{2} v^{3}$.

The meaning of the nondiagonal term $D_{21}^{(3)}$ can be more clear if it is presented as a function of the two output signals of the first stage

$$
D_{21}^{(3)}=2 a_{2} v_{\text {out1 }}^{(1)} v_{\text {out1 }}^{(2)} \text {, }
$$

where $v_{\text {out1 }}^{(1)}=K_{1} v$ is linear output signal, and $v_{\text {out1 }}^{(2)}=$ $a_{1} v^{2}=a_{1} v v$ is the second-order distortion signal produced by the first nonlinearity. Then we can interpret $D_{21}^{(3)}(52)$ as a result of mixing signals $v_{\text {out } 1}^{(1)}, v_{\text {out1 }}^{(2)}$ by the nonlinearity of the stage 2.

Here, the matrix $D^{(3)}$ is triangular because the circuit is unidirectional, but if feedback loop is present, then $D_{12}^{(3)} \neq 0$. To see this, consider the next example shown in Figure 2.

The transfer function $v_{\text {out }}(v)$ for the circuit is defined by the implicit expression $v_{\text {out }}(v)=v_{\text {out } 1}\left(v-v_{\text {out } 2}\left(v_{\text {out }}(v)\right)\right)$. After substituting (48) and (49) into this expression and equating terms of equal orders, one can obtain the following expressions for output linear signal, second- and third-order distortion contributions:

$$
\begin{gathered}
v_{\text {out } 1}^{(1)}=\frac{K_{1} v}{1+K_{1} K_{2}}, \\
D_{1}^{(2)}=\frac{a_{1} v^{2}}{\left(1+K_{1} K_{2}\right)^{3}}, \quad D_{2}^{(2)}=-\frac{a_{2} K_{1}^{3} v^{2}}{\left(1+K_{1} K_{2}\right)^{3}}, \\
D_{11}^{(3)}=\frac{-2 a_{1} D_{1}^{(2)} K_{2} v^{3}}{\left(1+K_{1} K_{2}\right)^{2}}+\frac{b_{1} v^{3}}{\left(1+K_{1} K_{2}\right)^{4}}, \\
D_{22}^{(3)}=\frac{-2 a_{2} D_{2}^{(2)} K_{1}^{2} K_{2} v^{3}}{\left(1+K_{1} K_{2}\right)^{2}}+\frac{-b_{2} K_{1}^{4} K_{2}^{3} v^{3}}{\left(1+K_{1} K_{2}\right)^{4}}, \\
D_{12}^{(3)}=\frac{2 a_{1} D_{2}^{(2)} v^{3}}{K_{1}\left(1+K_{1} K_{2}\right)^{2}}, \quad D_{21}^{(3)}=\frac{-2 a_{2} D_{1}^{(2)} K_{1}^{2} K_{2} v^{3}}{\left(1+K_{1} K_{2}\right)^{2}} .
\end{gathered}
$$

The similar generation of third-order distortions by the mixing of linear and second-order signals is present in any nonlinear circuit (as can be proved by Volterra series theory). So the third-order distortion contributions of the circuit can be characterized by the square matrix $D^{(3)}$ with entries $D_{n m}^{(3)}$.

The diagonal entry $D_{n n}^{(3)}$ defines the third-order signal that is produced in $n$th nonlinearity by the mixing of the linear signal with the second-order signal from the same nonlinearity (first term in (55)) and the mixing of linear signals by the third order coefficient of the nonlinearity (second term in (55)). The nondiagonal entry $D_{n m}^{(3)}$ defines the third-order signal that is produced in $n$th nonlinearity by the mixing of linear signals with the second-order signals produced in $m$ th nonlinearity.

The following properties of the contribution matrix exist.

(1) The sum of all matrix entries is equal to the total third-order distortion

$$
D_{\text {total }}^{(3)}=\sum_{n} \sum_{m} D_{n m}^{(3)}
$$

(2) If parameters of the $n$th nonlinearity are changed, then only entries of $n$th column and $n$th row are modified. All other entries remain unchanged. In particular, if parameters of the $n$th nonlinearity are set to zero, then all entries of $n$th column and $n$th row are zeros. All other entries remain unchanged.

(3) The sum of entries of $n$th column and $n$th row

$$
D_{n}^{(3)}=\sum_{m \neq n}\left(D_{n m}^{(3)}+D_{m n}^{(3)}\right)+D_{n n}^{(3)}
$$

defines the total influence of $n$th nonlinearity on the third-order distortion. It is equal to the difference between the total distortion and the distortion obtained without this nonlinearity. Note that value $D_{n}^{(3)}$ cannot be considered as the contribution of $n$th nonlinearity in the sense (46) because property (47) is not true. 
(4) If the circuit contains a group of nonlinearities $S=$ $\left\{i_{1}, i_{2}, \ldots\right\}$ and it is desired to present the group as one nonlinearity with index $k$, then the matrix is obtained using the following expressions:

$$
D_{k m}^{(3)}=\sum_{n \in S} D_{n m}^{(3)}, \quad D_{n k}^{(3)}=\sum_{m \in S} D_{n m}^{(3)}, \quad D_{k k}^{(3)}=\sum_{n \in S} \sum_{m \in S} D_{n m}^{(3)}
$$

Thus, the contribution matrix allows one to obtain various information on the influence of circuit nonlinearities upon the output nonlinear distortion.

The utility of the nondiagonal contribution entries can be illustrated by the following way. If the main contribution is defined by diagonal entry, then the overall circuit distortion can be improved by reducing of corresponding nonlinearity. But if such entry is nondiagonal one $D_{\mathrm{nm}}^{(3)}$, then a designer can also change frequency characteristics of the signal path from $m$ th to $n$th nonlinearity to attenuate the second-order signal through the path.

4.2. Evaluation of Contributions by Simplified Newton's Method. For the determination of individual contributions, the functional description of circuit model $f(z)$ (5) must be presented as the sum of nonlinear dependencies

$$
f(z)=\sum_{m} f^{(m)}(z)
$$

corresponding to the defined nonlinearities. The nonlinear dependencies can be, for example, currents of circuit components or individual terminal currents or even separate capacitance and DC currents. The representation (60) is provided in circuit simulators at the step of equation formulation from models of individual nonlinearities, with interconnection equations obtained by using Kirchhoff's current law.

In accordance with (60), the Jacobian matrix of (5) also can be presented as the sum of corresponding matrices of the partial derivatives

$$
J(z)=\sum_{m} J^{(m)}(z), \quad J^{(m)}(z)=\frac{\partial f^{(m)}(z)}{\partial z}
$$

For the representations (60), (61), step 2 (12) can be written in the form

$$
J\left(z^{(0)}\right) \Delta z^{(1)}=\sum_{m}\left(J^{(m)}\left(z^{(0)}\right) \Delta z^{(0)}-f^{(m)}\left(z^{(1)}\right)\right) .
$$

Here, we exploit the fact that from (11), we have

$$
\sum_{m}\left(J^{(m)}\left(z^{(0)}\right) \Delta z^{(0)}\right)=J\left(z^{(0)}\right) \Delta z^{(0)}=w .
$$

Each term in the residual vector of (62) defines the effect of $m$ th nonlinearity because it presents the difference between the linearized $J^{(m)}\left(z^{(0)}\right) \Delta z^{(0)}$ and the nonlinear $f^{(m)}\left(z^{(1)}\right)$ dependencies of the nonlinearity. Hence, the second-order distortion can be written as a sum of individual contributions

$$
\begin{gathered}
\Delta z^{(1)}=\sum_{m} \Delta z^{(1, m)} \\
\Delta z^{(1, m)}=J^{-1}\left(z^{(0)}\right)\left(J^{(m)}\left(z^{(0)}\right) \Delta z^{(0)}-f^{(m)}\left(z^{(1)}\right)\right) .
\end{gathered}
$$

To obtain third-order distortion as the sum of individual contributions, we use a modified expression (16) for step 3. After substituting (62), (64) into (16), we obtain

$$
J\left(z^{(0)}\right) \Delta z^{(2)}=\sum_{n}\left(J^{(n)}\left(z^{(0)}\right)-J^{(n)}\left(z^{(1)}\right)\right) \sum_{m} \Delta z^{(1, m)} .
$$

Thus, the third-order distortion is

$$
\begin{gathered}
\Delta z^{(2)}=\sum_{n} \sum_{m} \Delta z^{(2, n, m)}, \\
\Delta z^{(2, n, m)}=J^{-1}\left(z^{(0)}\right)\left(J^{(n)}\left(z^{(0)}\right)-J^{(n)}\left(z^{(1)}\right)\right) \Delta z^{(1, m)} .
\end{gathered}
$$

Each vector (66) depends only on two nonlinearities $m, n$.

Usually the distortion at the output node is of interest. The components of vectors (64) corresponding to the output node form a vector of second-order contributions $D_{m}^{(2)}=\Delta z_{\text {out }}^{(1, m)}$, and corresponding components of the vectors (66) form the matrix of third-order contributions $D_{n m}^{(3)}=$ $\Delta z_{\text {out }}^{(2, n), m}$, where subscript out denotes the output node.

The algorithm for obtaining individual contributions is based on expressions (64) and (66), which may be represented in the frequency domain (in accordance with (34) and (37)) as

$$
\begin{gathered}
J^{\mathrm{SS}}\left(v_{j}^{(2)}\right) \Delta X^{(1, m)}\left(v_{j}^{(2)}\right)=-H^{(1, m)}\left(v_{j}^{(2)}\right), \\
J^{\mathrm{SS}}\left(v_{j}^{(3)}\right) \Delta X^{(2, n, m)}\left(v_{j}^{(3)}\right)=-H^{(2, n, m)}\left(v_{j}^{(3)}\right)-\delta_{n}^{m} H^{(1, n)}\left(v_{j}^{(3)}\right),
\end{gathered}
$$

where $\delta_{n}^{m}$ is the Kronecker delta. In accordance with (26), $H^{(2, n, m)}=\left[J^{\mathrm{HB}(n)}\left(X^{(1)}\right)-J^{\mathrm{HB}(n)}(\tilde{X})\right] \Delta X^{(1, m)}$.

The computations can be performed in the same numerical framework presented in Section 3. To obtain vectors $H^{(1, m)}\left(X^{(1)}\right)$ and matrices $J^{\mathrm{HB}(n)}\left(X^{(1)}\right)$, the $\mathrm{HB}$ loading procedure must be developed on the per-nonlinearity basis.

Let $N_{N}$ be the number of nonlinearities in the circuit, and $K_{2}, K_{3}$ are the numbers of second- and third-order small signal frequencies, respectively. Then the computation of second-order contributions requires solving $K_{2} N_{N}$ linear systems (67) with only $K_{2}$ different matrices. For the thirdorder contribution matrix $K_{3} N_{N}^{2}$ linear systems (68) should be solved. Fortunately, using the technique of adjoint linear system, this work is reduced to solving $K_{3}$ linear system with transposed matrix and $K_{3} N_{N}^{2}$ computations of dot products.

\section{Numerical Results}

5.1. Comparison with Harmonic Balance. We compare our new approach for the periodic distortion analysis based on 


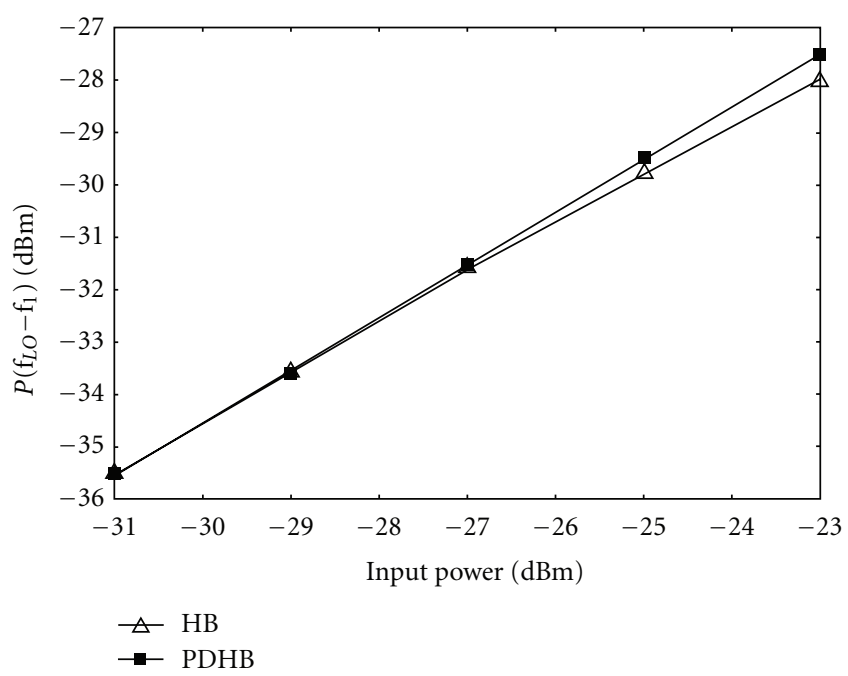

Figure 3: Output power of frequency component $\left(f_{\mathrm{LO}}-f_{1}\right)$ versus input power.

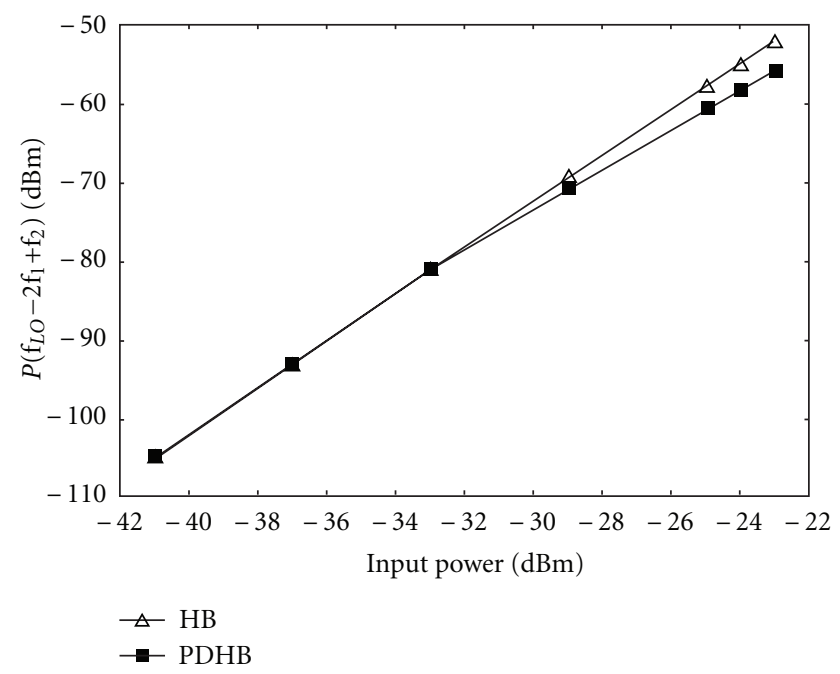

FIgURe 4: Output power of frequency component $\left(f_{\mathrm{LO}}-2 f_{1}+f_{2}\right)$ versus input power.

HB method (PDHB) with complete multitone HB analysis. Numerical experiments are performed for two MOSFET mixers. The first circuit is the single-balanced mixer containing 3 MOS transistors. The first mixer has input parameters $A_{\mathrm{LO}}=2.5 \mathrm{~V}, f_{\mathrm{LO}}=375 \mathrm{MHz}, f_{1}=394.9 \mathrm{MHz}$, and $f_{2}=$ $395.1 \mathrm{MHz}$, with $r f$ input varying from 0 to $0.5 \mathrm{~V}$. The second circuit is the double-balanced mixer [20] containing 6 MOS transistors and biasing circuitry. The second mixer has parameters $A_{\mathrm{LO}}=0.75 \mathrm{~V}, f_{\mathrm{LO}}=985 \mathrm{MHz}, f_{1}=899 \mathrm{MHz}$, and $f_{2}=901 \mathrm{MHz}$, with $r f$ input varying from 0 to $0.15 \mathrm{~V}$. In both circuits, $f_{1}$ and $f_{2}$ are treated as small as signals in the PDHB analysis, whereas the HB analysis must be a full 3-tone simulation.

The computed dependencies of the output power of the frequency component $\left(f_{\mathrm{LO}}-f_{1}\right)$ on the input power are shown in Figure 3 for the first mixer. The obtained curves are

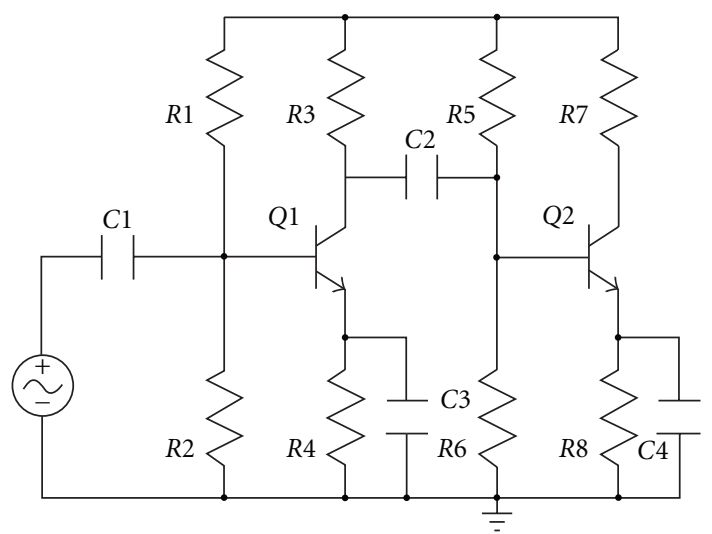

Figure 5: Two-stage bipolar amplifier.

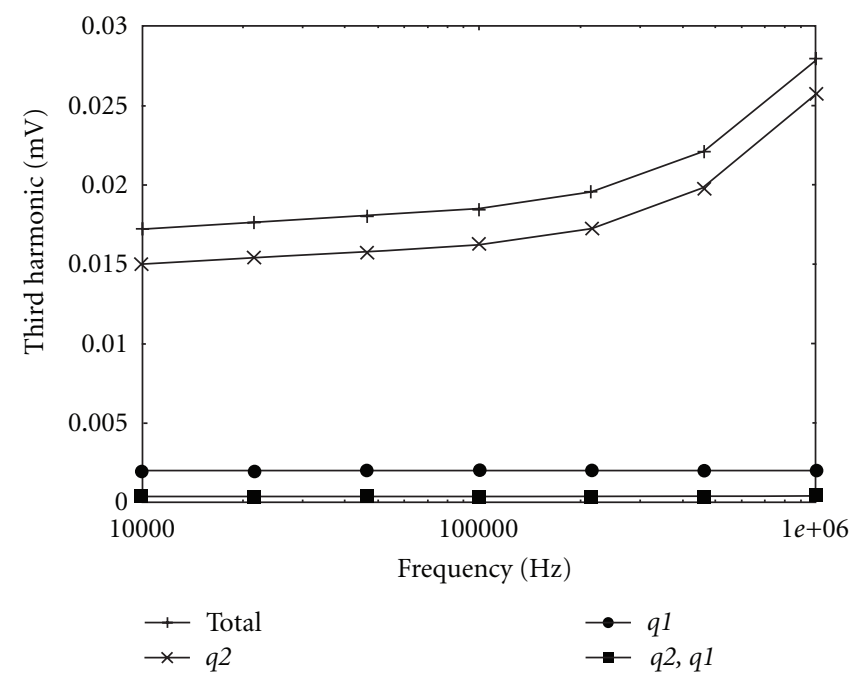

FIGURE 6: Third harmonic and its contributions.

in good agreement, especially for small $r f$ input amplitudes as expected. Similar results are obtained for the second mixer. The dependencies of the output power at the third-order intermodulation product $\left(f_{\mathrm{LO}}-2 f_{1}+f_{2}\right)$ are shown in Figure 4 for the first mixer. The obtained curves are in good agreement for practical input amplitudes.

The computational efficiency of the PDHB approach for the two mixer circuits can be seen from Table 1 . The number of $\mathrm{HB}$ harmonics for the three tones $f_{\mathrm{LO}}, f_{1}, f_{2}$ is $(15,3,3)$ and $(7,3,3)$ for the first and second mixer, respectively. The corresponding orders of linear systems for the two different methods are given in columns 3,4 . The number of linear systems to be solved is 8 and 18 in $\mathrm{HB}$ and PDHB analyses, respectively, for the first example. The second mixer requires solving 9 and 19 linear systems. In spite of increased number of linear systems, the CPU time is less in PDHB analysis due to the much smaller order of systems.

5.2. Examples of Individual Contributions. The first example is the two-stage bipolar amplifier shown in Figure 5. The distortion analysis is applied to compute second- and 


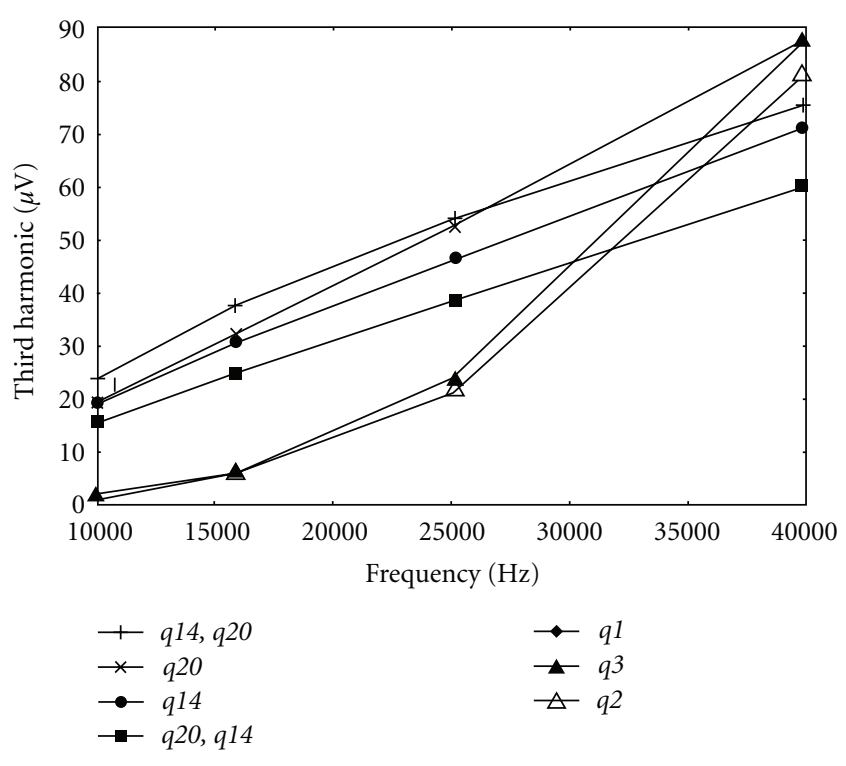

Figure 7: Contributions to the third harmonic.

TABle 1

\begin{tabular}{lccccc}
\hline \multicolumn{2}{c}{ Circuit } & \multicolumn{2}{c}{ Linear system order } & \multicolumn{2}{c}{ CPU time, sec } \\
\hline Number & $N$ & HB & PDHB & HB & PDHB \\
\hline 1 & 30 & 91140 & 1860 & 360 & 13 \\
2 & 50 & 79380 & 1500 & 399 & 15 \\
\hline
\end{tabular}

third-order harmonic distortion. Figure 6 shows the third harmonic together with contributions from different transistors as a function of frequency. It is seen from the figure that the most dominant contribution is due to the transistor of the output stage. The contribution of the first-stage transistor $q 1$ is smaller by a factor of $8-12$, and the contribution due to the interaction of transistors $q 1$ and $q 2$ is negligible in this simple example. This relation is changed with introducing feedback loop in the amplifier.

The second example is an OpAmp UA741 [24]. The transistors $q 1, q 2, q 3$ belong to the input stage of the amplifier, and transistors $q 14, q 20$ are from the output stage. Figure 7 shows the dominant contributions related with these transistors to third harmonic as a function of frequency. Note that contributions of the output stage transistors are dominated over input stage transistors at low frequencies while they become the same order at high frequencies. Also note that contributions due to interaction between transistors $q 14, q 20$ are comparable with contributions of $q 14$ and $q 20$ individually and therefore cannot be neglected in this example.

\section{Conclusion}

A new approach for nonlinear distortion analysis has been presented. This approach does not require the computation of second and third derivatives of the semiconductor device models and, thus, has an important advantage over the Volterra series-based distortion analysis. The new technique is applicable to the class of weakly nonlinear and periodic time-varying circuits.

A computational algorithm for periodic distortion analysis of communication circuits has been developed. Three steps of the algorithm provide the same order of accuracy that three steps of the distortion procedure based on Volterra series methods.

In comparison with complete nonlinear steady-state analysis (such as harmonic balance), the approach provides significant reducing of computational efforts.

In addition, the new technique allows computing the contributions of individual circuit components to the distortion. A matrix form for the characterization of third-order individual distortion contributions has been proposed. The numerical procedures for computing individual contributions have been developed, which provide designers with valuable information on the influence of various nonlinearities upon the total distortion.

\section{References}

[1] K. Kundert, "Introduction to RF simulation and its application," IEEE Journal of Solid-State Circuits, vol. 34, no. 9, pp. 1298-1319, 1999.

[2] M. B. Steer, J. W. Bandler, and C. M. Snowden, "Computeraided design of RF and microwave circuits and systems," IEEE Transactions on Microwave Theory and Techniques, vol. 50, no. 3, pp. 996-1005, 2002.

[3] P. Wambacq and W. Sansen, Distortion Analysis of Analog Integrated Circuits, Kluwer Academic, Norwell, Mass, USA, 1998.

[4] S. A. Maas, Nonlinear Microwave Circuits, Artech House, Norwood, Mass, USA, 1998.

[5] K. S. Kundert, J. White, and A. Sangiovanni-Vincentelli, Steady-State Methods for Simulating Analog and Microwave Circuits, Kluwer Academic, Boston, Mass, USA, 1990.

[6] P. Feldmann, B. Melville, and D. Long, "Efficient frequency domain analysis of large nonlinear analog circuits," in Proceedings of the IEEE Custom Integrated Circuits Conference, pp. 461464, 1996.

[7] V. Rizzoli and A. Neri, "State of the art and present trends in nonlinear microwave CAD techniques," IEEE Transactions on Microwave Theory and Techniques, vol. 36, no. 2, pp. 343-365, 1988.

[8] R. Gilmore and M. B. Steer, "Nonlinear circuit analysis using the method of harmonic balance-a review of the art. Part I. Introductory concepts ," International Journal of Microwave and Millimeter-Wave Computer-Aided Engineering, vol. 1, no. 1, pp. 22-37, 1991.

[9] R. Gilmore and M. B. Steer, "Nonlinear circuit analysis using the method of harmonic balance-a review of the art. II. Advanced concepts," International Journal of Microwave and Millimeter-Wave Computer-Aided Engineering, vol. 1, no. 2, pp. 159-180, 1991.

[10] M. S. Nakhla and J. Vlach, "A piecewise harmonic-balance technique for determination of periodic response of nonlinear systems," IEEE Transactions on Circuits and Systems, vol. 23, no. 2, pp. 85-91, 1976.

[11] A. Ushida and L. Chua, "Frequency-domain analysis of nonlinear circuits driven by multi-tone signals," IEEE Transactions on Circuits and Systems, vol. 31, no. 9, pp. 766-778, 1984. 
[12] J. Chen, D. Feng, J. Phillips, and K. Kundert, "Simulation and modeling of intermodulation distortion in communication circuits," in Proceedings of the IEEE Annual Custom Integrated Circuits Conference, pp. 5-8, 1999.

[13] S. Narayanan, "Transistor distortion analysis using Volterra series representations," Bell System Technical Journal, vol. 46, no. 5, pp. 991-1024, 1967.

[14] Y. L. Kuo, "Distortion analysis of bipolar transistor circuits," IEEE Transactions on Circuit Theory, vol. 20, no. 6, pp. 709$716,1973$.

[15] S. Chisholm and L. Nagel, "Efficient computer simulation of distortion in electronic circuits," IEEE Transactions on Circuit Theory, vol. 20, no. 6, pp. 742-745, 1973.

[16] P. Dobrovolny, G. Vandersteen, P. Wambacq, and S. Donnay, "Analysis and white-box modeling of weakly nonlinear timevarying circuits," in Proceedings of the Design, Automation and Test in Europe Conference, pp. 624-629, 2003.

[17] T. Yuan and A. Opal, "Distortion analysis of periodically switched nonlinear circuits using time-varying Volterra series," IEEE Transactions on Circuits and Systems I, vol. 48, no. 6, pp. 726-738, 2001.

[18] M. M. Gourary, S. G. Rusakov, S. L. Ulyanov, M. M. Zharov, K. K. Gullapalli, and B. J. Mulvaney, "New computational technique for periodic distortion analysis of communication circuits," in Proceedings of the IEEE MITT-S International Microwave Symposium Digest Conference, vol. 2, pp. 941-944, 2004.

[19] J. M. Ortega and W. C. Rheinboldt, Iterative Solution of Nonlinear Equations in Several Variables, Academic Press, 1970.

[20] P. Li and L. Pileggi, "Efficient per-nonlinearity distortion analysis for analog and RF circuits," IEEE Transactions on Computer-Aided Design of Integrated Circuits and Systems, vol. 22, no. 10, pp. 1297-1309, 2003.

[21] J. F. Traub, Iterative Methods for the Solution of Equations, Chelsia, 1982.

[22] M. M. Gourary, S. G. Rusakov, S. L. Ulyanov, M. M. Zharov, and B. J. Mulvaney, "A new simulation technique for periodic small-signal analysis," in Proceedings of the Conference on Design, Automation and Test in Europe, pp. 244-249, Munich, Germany, march 2003.

[23] M. Okumura, T. Sugawara, and H. Tanimoto, "An efficient small signal frequency analysis method of nonlinear circuits with two frequency excitations," IEEE Transactions on Computer-Aided Design of Integrated Circuits and Systems, vol. 9, no. 3, pp. 225-235, 1990.

[24] A. Vladimirescu, The Spice Book, John Wiley, New York, NY, USA, 1994. 

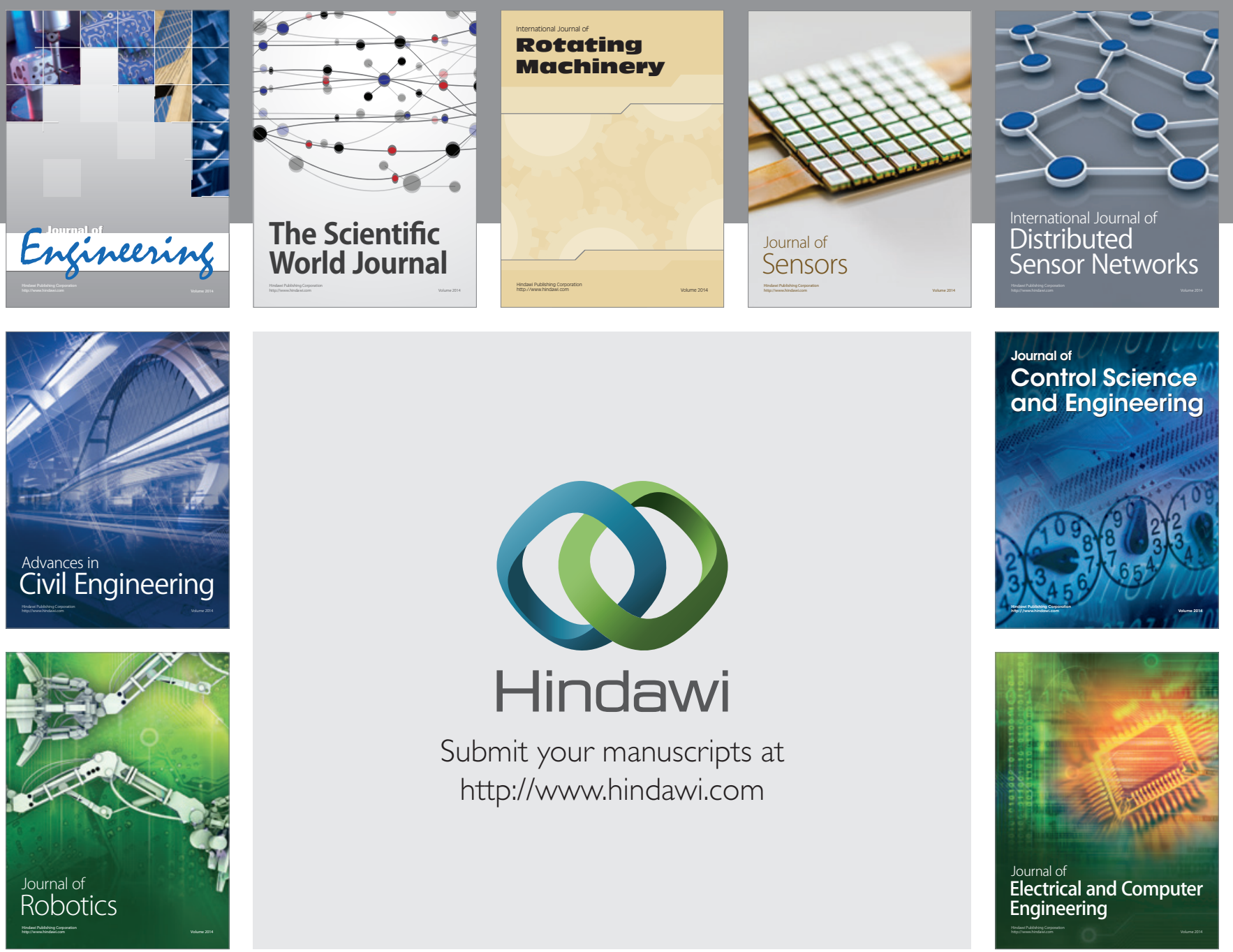

Submit your manuscripts at

http://www.hindawi.com
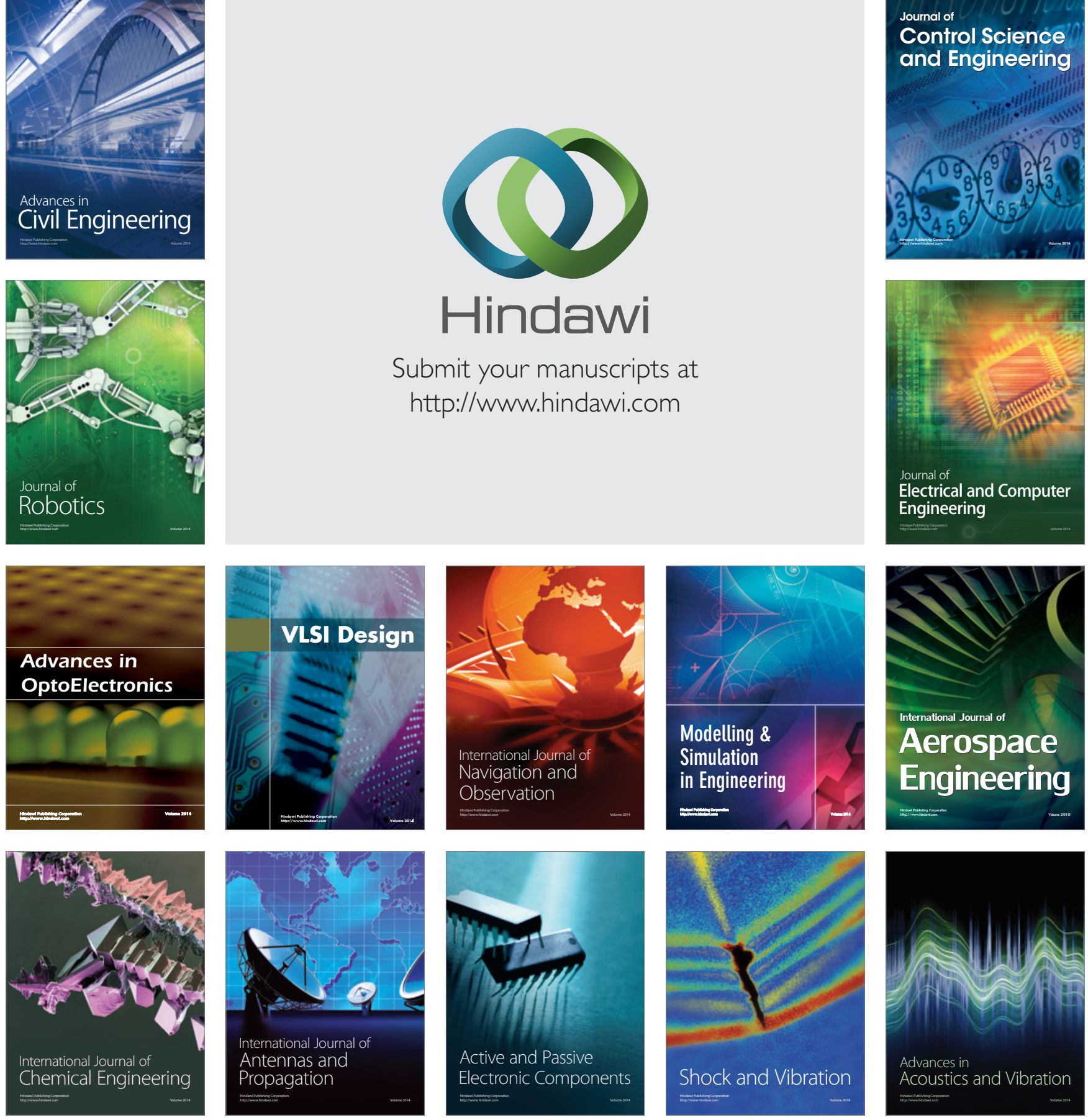